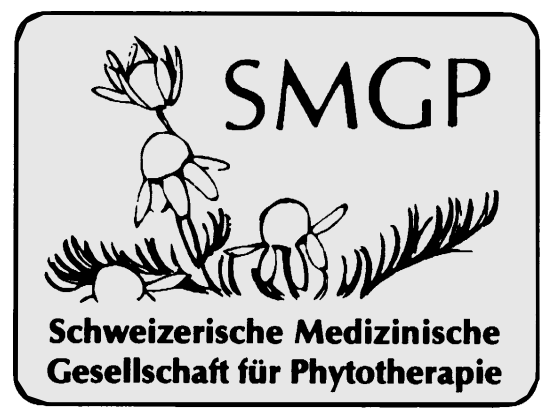

\section{Union schweizerischer komplementärmedizinischer Ärzteorganisationen}

\section{Jahre Standes- und Gesundheitspolitik: Rück- und Ausblick}

Am 26. April feierte die Union komplementärmedizinischer Ärzteorganisationen in Olten in kleinem Kreis mit den Delegierten und den Verbandspräsidenten respektive deren Vertretern ihren 5. Geburtstag. Anlässlich seines Jubiläumsreferats strich Unionssekretär Max Küenzi heraus, dass bereits lange vor der Unionsgründung verschiedene Gruppierungen sich in verdankens- und lobenswerter Art und Weise für die ärztliche Komplementärmedizin eingesetzt haben. $\mathrm{Zu}$ erwähnen sind vorab die Unionsmitglieder selber, die ja auf eine viel längere Geschichte als die UNION zurückblicken können. Speziell hervorzuheben ist die Schweizerische Ärztegesellschaft für Erfahrungsmedizin (SAGEM), die im damaligen Vereinigungsprozess eine wichtige Stellung eingenommen hat. $\mathrm{Zu}$ erwähnen ist im Weiteren das Forum für Ganzheitsmedizin, das mit dem Ergreifen des Referendums gegen das neue Krankenversicherungsgesetz ein «Verhandlungspfand» erkämpft hat, sowie die Kollegiale Instanz für Komplementärmedizin an der Medizinischen Fakultät der Universität Bern und der Lehrstuhl für Naturheilkunde an der Universität. $\mathrm{Zu}$ nennen sind zudem eine Vielzahl von Persönlichkeiten, die nebenbei und ehrenamtlich mit grossem Einsatz mitgeholfen haben, der ärztlichen Komplementärmedizin zu der ihr gebührenden Stellung zu verhelfen. Der Unionssekretär sprach allen diesen Vorkämpfern und Vorkämpferinnen seinen Dank aus. Im zweiten Teil des Referats wurde die Unionsgeschichte von einer verbandspolitischen Warte aus gewürdigt (separater Text).

Zum Abschluss wies Max Küenzi auf die Tagesaktualität hin. Insbesondere zitierte er die Kritik, die die UNION im verbandseigenen Mitteilungsorgan am neuen Heilmittelgesetz angebracht hatte. Die UNION

Gesellschaftsmitteilungen · Society Bulletins

Forsch Komplementärmed Klass Naturheilkd 2001;8:239-240

bemängelte vor allem das «unbernische» Tempo, mit dem das Gesetz und vor allem dessen Verordnungen in Kraft treten sollten. Bekanntlich wurde kurz vor dem Jubiläum beschlossen, das In-Kraft-Treten des neuen Gesetzeswerks auf den 1. Januar 2002 zu verschieben. Diese Meldung führte direkt zur Wortübergabe an Frau Dr. Karoline Mathys, die Leiterin der Abteilung Komplementär- und Phytoarzneimittel der IKS und des nachmaligen Schweizerischen Heilmittelinstituts, die als gern gesehener Gast unter der kleinen Jubiläumsschar weilte. Die Referentin verstand es geschickt und mit Sachlichkeit, das neue Gesetz und dessen Verordnungen zu erläutern. Während die einen damit beruhigt werden konnten, verblieb bei den anderen nach wie vor eine gewisse Skepsis. Von Wichtigkeit ist jedoch, dass das Gespräch auf einer vertrauensvollen und konstruktiven Basis aufgenommen werden konnte - und dies dürfte beim Jubiläum der UNION und am nachfolgenden ungezwungenen Apéro gelungen sein.

Anmerkung: Die SMGP zählt zu den Gründungsmitgliedern der UNION.

Verbände-braucht es die überhaupt noch?

\section{Max Künzi}

Zum Autor: Lic. iur. Max Küenzi, Sekretär der UNION, befasste sich in den Jahren 1993 bis 1996 mit der Thematik «Komplementärmedizin und Gesundheitsrecht» im Rahmen eines Projekts des Schweizerischen Nationalfonds, das seinen Niederschlag in einer Publikation der Reihe "Neue Literatur zum Recht» im Verlag Helbing \& Lichtenhahn fand. Anschliessend betreute er die Vorarbeiten zur Unionsgründung und ab Mai 1996 im Teilzeit-Mandat das Unionssekretariat.

Diese provokative Frage wurde in der Neuen Zürcher Zeitung (NZZ) im Zusammenhang mit der Entwicklung der schweizerischen Wirtschaftsverbände gestellt. Verbände sind aufgrund unseres direktdemokratischen Systems ein wichtiges und nicht mehr wegzudenkendes Instrument zur sachlichen Interessenvertretung im politischen Meinungsbildungsprozess. Verbände haben dann eine Daseinsberechtigung, wenn es ihnen gelingt, auf der politischen Ebene wirkungsvoll $\mathrm{zu}$ agieren. Die Legitimation der UNION gründet historisch gesehen unter anderem auf dem Wunsch der FMH, einen einzigen Ansprechpartner in Sachen der ärztlichen Komplementärmedizin zu haben. Relativ rasch nach der Unionsgründung wurde die
UNION in die FMH-Statuten als mitspracheberechtigte Ärzteorganisation aufgenommen. Die UNION hat heute in der Ärztekammer Diskussions- und Antragsrecht - und in der Konferenz der Präsidenten der Fachgesellschaften Diskussions-, Antrags- und Stimmrecht. In der UNION ist man der Auffassung, dass eine wirkungsvolle Standespolitik und Verbandsarbeit ein gutes Einvernehmen und eine gute Kooperation mit der FMH voraussetzt. So geht etwa auch das Bundesamt für Sozialversicherung davon aus, dass Nominationen für die eidgenössischen Kommissionen (Leistungskommission, Arzneimittelkommission), soweit sie die Ärzteschaft betreffen, gemeinsam vorgenommen werden. Der statutarische Auftrag der UNION ist daneben unmissverständlich: Die UNION hat die standespolitischen Interessen der ärztlichen Komplementärmedizin wahrzunehmen. Dieser Grundsatz wird in den Leitsätzen der UNION konkretisiert. Stichworte dazu sind: Tarife, Qualität, Gleichberechtigung der Komplementärmedizin in Praxis, Lehre und Forschung, Öffentlichkeitsarbeit.

Als Meilenstein in der Unionsarbeit kann die Aufnahme der Akupunktur/TCM, der Anthroposophischen Medizin, der Klassischen Homöopathie, der Neuraltherapie und der Phytotherapie in die Grundversicherung bezeichnet werden. Die Aufnahme erfolgte allerdings unter dem Vorbehalt, dass im Zeitraum zwischen dem 1. Juli 1999 und dem 30. Juni 2005 eine Evaluation durchgeführt wird - von der Evaluationspflicht ausgenommen ist die Akupunktur. Diese Evaluation gilt es, wissenschaftlich und gesundheitspolitisch sorgfältig zu begleiten. Das gleiche gilt übrigens für den Bereich der Tarife und des Tarmed: Die Gleichstellung der ärztlichen Komplementärmedizin mit den übrigen Grundversorgern konnte erreicht werden - damit ist vorerst aber nur ein Minimalziel erreicht. So hat sich etwa herausgestellt, dass die Einstufung der Neuraltherapie geändert werden muss. Es stellt sich auch die Frage, ob die komplementärmedizinischen Fähigkeitsausweise nicht tariflich besser berücksichtigt werden müssten.

Ein weiterer Meilenstein in der Unionsarbeit war sicher der mit grossem Erfolg durchgeführte erste Unionskongress in den Räumlichkeiten des Universitätsspitals in Bern. Die Vorbereitungsarbeiten für den Kongress 2002 laufen demnächst an - er findet am 13./14. September 2002 wiederum in Bern am Universitätsspital statt. Im Übrigen ist der Kongress 2004 ebenfalls bereits fest für den 10./11. September «gebucht». Die Kongresse 2002 und 2004

\section{KARGER}

Fax +497614520714

E-mail Information@Karger.de

www.karger.com 
werden im Zeichen der laufenden Evaluation stehen - bekanntlich läuft diese voraussichtlich am 30. Juni 2005 ab.

Abschliessend soll noch kurz auf weitere Unionsprojekte, die von Wichtigkeit sind, hingewiesen werden: Im Frühherbst dieses Jahres wird eine Publikation unter dem Titel «Komplementärmedizin und Krankenversicherung» herausgegeben. In diesem Wegweiser wird die ärztliche Komplementärmedizin und deren Umfeld auf verständliche Art und Weise dargestellt. Auch noch in diesem Jahr soll hoffentlich die Forschungsstiftung Komplementärmedizin aus der Taufe gehoben werden. Bekanntlich hat die UNION beschlossen, für die Förderung der Forschung im Bereich der ärztlichen Komplementärmedizin eine Stiftung zu gründen. Diese Stiftung soll mit einem Stiftungskapital von CHF 50000,dotiert und dann auf dem Weg des FundRaising mit weiteren Mitteln ausgestattet werden. Dank der grosszügigen Unterstützung einer befreundeten Organisation konnte innert Kürze die Hälfte des Stiftungskapitals bereits beigebracht werden. Schliesslich wurde eine Ombudsstelle für die ärztliche Komplementärmedizin ins Leben gerufen und die Genehmigung eines unionseigenen Fertigkeitsausweises für die Disziplinen, die nicht der Grundversicherung unterstellt sind, sollte demnächst erfolgen können.

Die Frage der NZZ kann beantwortet werden: Es braucht Verbände wie die Union, denn wo stünde die Komplementärmedizin ohne deren Einsatz?

Beat Meier, Präsident SMGP

Leitlinien der Ombudsstelle Komplementärmedizin der Union

\section{Grundsätzliches}

Die Ombudsstelle der UNION berät Privatpersonen (Ärzte/Ärztinnen und weitere Medizinalpersonen, Patienten/Patientinnen) ausschliesslich im Bereich der ärztlichen Komplementärmedizin und Krankenversicherung (Sozialversicherung und Privatversicherung). Die Ombudsstelle strebt Mediationslösungen und einvernehmliche Lösungen an.

\section{Praktisches}

Die Ombudsstelle erteilt eine Erstauskunft kostenlos. Für weitergehende Bearbeitungen kann im gegenseitigen Einvernehmen zu einem reduzierten Ansatz eine Rechnung gestellt werden. Die Ombudsstelle läuft über die Mediationspraxis Lic.iur. Max Künzi.

Anschrift: Ombudsstelle der UNION,

Schachenstrasse 21,

CH-4702 Oensingen,

Tel. +41 62 396-29 02, Fax -24 10,

E-mailinfo@unioncomed.ch.
3. Vorgehen

Damit die Ombudsstelle eine konkrete Prüfung vornehmen kann, benötigt sie eine kurze Darstellung des Sachverhalts und

die vollständigen Akten. Kurze Auskünfte können telefonisch erteilt werden. Sprechstunden gibt es keine.

\section{Schweizerische Jahrestagung für Phytotherapie 15. November 2001 in Baden Vorläufiges Programm, Stand: 2. 7. 2001}

Phytotherapie in der Pädiatrie

\begin{tabular}{llll}
\hline Zeit & Thema/Arbeitstitel & Referent & Sponsor \\
\hline $09.10-09.15$ & Begrüssung & Prof. Dr. Beat Meier & \\
\hline 09.15-09.45 & $\begin{array}{l}\text { Chairman-Morgensession } \\
\text { Probleme des Kinderarztes im heutigen Umfeld } \\
\text { und Erwartungen an die Komplementärmedizin, } \\
\text { insbesondere die Phytotherapie }\end{array}$ & $\begin{array}{l}\text { Prof. Dr. Beat Meier } \\
\text { Dr. Andreas Tschumi, }\end{array}$ & Kinderarzt, Schaffhausen
\end{tabular}

09.45-10.15 Die Dosierungsfrage bei Kindern in der Phytotherapie

Michael Elosge, Essenheim offen

10.15-10.30 Diskussion Referate 1 und 2

10.30-11.00 Pause/Ausstellung

11.00-11.30 Nachtkerzenöl bei Kindern - eine Hoffnung bei Dr. Reto Rupf Sidroga AG atopischer Dermatitis?

11.30-12.00 Therapiekonzepte bei Allergien im Kindesalter Dr. Andreas Schapowal, offen

12.00-12.15 Diskussion Referate 3 und 4

12.15-12.30 Verleihung der SMGP-Zertifikate $2001 \quad$ Prof. Dr. Beat Meier $\quad$ SMGP

$\begin{array}{lll}\text { Verleihung des Alfred-Vogel-Preises } 2001 & \text { Frau D. Vogel, Roggwil } \quad \text { Bioforce AG }\end{array}$

12.30-14.00 Mittagspause/Stehlunch/Ausstellung

14.00-15.15 Workshop: Moderation: Spagyros AG

Hyperkinetische Verhaltensstörungen bei Dr. Beatrice Falch, Zürich Parsenn-

Kindern - Ritalin versus Phytotherapie. Teilnehmer: Produkte

Dr. Heinrich R. Otremba,

St.Gallen;

Dr. Barbara Kask;

Dr. Heiner Frei;

NN

Chairwoman-Nachmittagssession PD Dr. Ursula von Mandach,

Zürich

14.00-14.30 Verträglichkeit von pflanzlichen Arzneimitteln Dr. Klemens Brühwiler, offen bei Kindern - Beobachtungen aus der Praxis Elgg

14.30-15.00 Alkohol in Arzneimitteln: Wie viel ist bei Kindern vertretbar?

Prof. Dr. Bernhard offen Lauterburg, Bern

15.00-15.15 Diskussion Referate 5 und 6

15.15-15.45 Pause/Ausstellung

15.45-16.45 Erfahrungen mit dem Einsatz pflanzlicher Sedativa bei Kindern und Jugendlichen

Passionsblume/Weissdorn in Kombination Dr. S. Büechi, Trogen Parsenn-Produkte, Küblis

Baldrian und/oder Johanniskraut Dr. M. Mannel, Berlin Medichemie Bioline

Johanniskraut/Baldrian-Hopfen in Kombination Dr. Josef Laimbacher， Zeller Medical St. Gallen

Diskussion

\begin{tabular}{lll}
\hline $16.45-17.00$ & $\begin{array}{l}\text { Bericht aus dem Workshop } \\
\text { Bilanz der Tagung }\end{array}$ & $\begin{array}{l}\text { offen } \\
\text { Prof. Dr. B. Meier }\end{array}$ \\
\hline 17.15 & Jahresversammlung SMGP & \\
\hline
\end{tabular}

